\title{
Spin and Gradient Echoes
}

\section{OVERVIEW}

\section{The Basic Signal Behavior}

Up to this point, we have discussed the basic building blocks of creating a signal and being able to measure it. In this unit, we introduce the concept of an echo. The phase of the spins plays the fundamental role in this process. We begin with a general description as to how an echo occurs and then discuss both spin echoes and gradient echoes. This also gives us an important piece of preliminary information which will be necessary for the further discussion of one-dimensional imaging in UNIT B4.2.

Consider first the magnetization $M_{\mathrm{y}}$ immediately after it has been created. It will precess about the main field clockwise when looking from the top. The signal also decays and will behave in the laboratory frame (see Fig. B4.1.1A) according to:

$$
S=S_{0} \cos (\omega t) \exp \left(-t / T_{2}\right)
$$

where $\omega$ is the angular frequency and after demodulation (hence, in the rotating frame, see Fig. B4.1.1B) according to

$$
S=S_{0} \exp \left(-t / T_{2}\right)
$$

This simple exponential decay represents the basic temporal dependence of the signal.

The basic assumption we have made so far is that the background static field is perfectly homogeneous. Practically, this is not true and so the question is, "How does a locally inhomogeneous field affect the signal?" Well, $T_{2}$ decay itself is already a partial answer to this, but it comes from irreversible effects (see UNIT B3.1). One can simply guess that there will be a similar signal loss with $T_{2}$ being replaced by $T_{2}{ }^{*}$ and $T_{2}{ }^{*}$ smaller than $T_{2}$. This can be expressed as:

$$
S=S_{0} \exp \left(-t / T_{2}^{*}\right)
$$

with:

$$
1 / T_{2}^{*}=1 / T_{2}+|\gamma \Delta B|
$$

where $\Delta B$ represents the local field inhomogeneity across a pixel and $\gamma$ is the gyromagnetic ratio. Although this extra signal loss is not generally exponential, the signal loss estimated from such an approximation is a good back-of-the-envelope calculation of the expected effect. The take home point is that the presence of the inhomogeneity causes the signal to decay faster. 
A

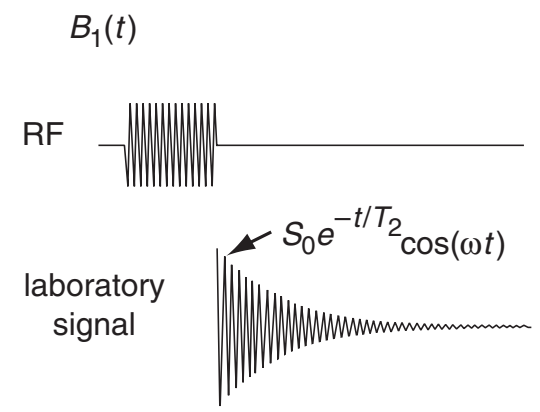

C
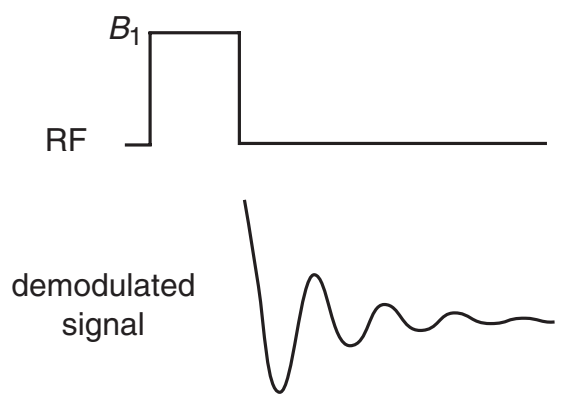

B
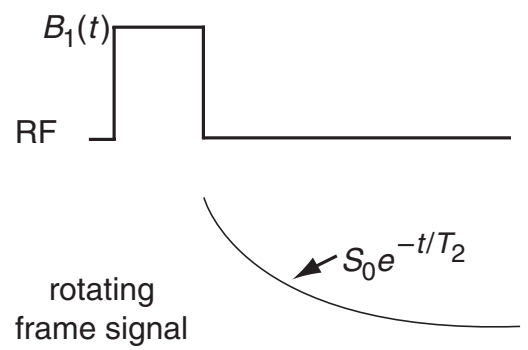

frame signal

D
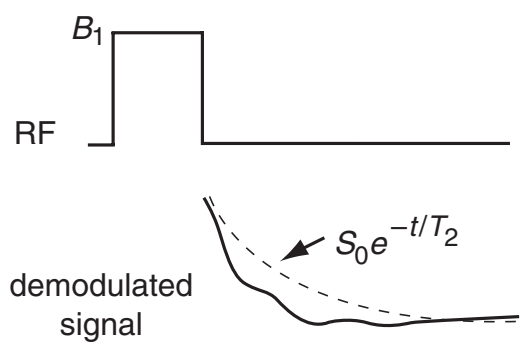

Figure B4.1.1 (A) The FID signal in the laboratory frame for all spins precessing at the same Larmor frequency. The laboratory RF transmit field oscillates at that frequency. (B) The same experiment but as measured in the Larmor rotating frame (i.e., demodulated). The rotating frame RF field is at "rest." (C) The demodulated FID signal when the demodulation is not exactly at the Larmor frequency. (D) The total demodulated FID signal from several isochromats, each with slightly different Larmor frequencies, exhibiting a decay, with slow oscillations, that is faster than $T_{2}$ decay alone, due to dephasing. The demodulation in (D) is determined by the average (fast) frequency. Note that the laboratory signal in $(A)$ is only suggestive since the oscillations seen in practice are too rapid to display. The $T_{2}^{\prime}$ effects have not been included in any of the curves; note that the differences in case (D) could be considered, alternatively, to be due to field inhomogeneities.

\section{The Basic Concept of an Echo and Dephasing}

One of the most frequently used terms in the MR field is the "echo." The echo is one of the building blocks and fundamental concepts in MR imaging. It represents the point where all the spins in the volume of interest have the same phase. When an echo occurs, the spins are referred to as being in-phase, or refocused. Since the MR signal is governed by the vector sum of all the available magnetic moments per unit volume, it is clear that the maximum MR signal is obtained when all the spins in the volume of interest have the same phase-i.e., the total magnetization per unit volume is:

$$
\vec{M}=\frac{1}{V} \sum_{i} \vec{M}_{i}
$$

where $V$ is the imaging volume and $\vec{M}_{i}$ is the local spin isochromat magnetization. 
Imagine a set of spins all starting off in synchrony at the time $t=0$. If they each see a different magnetic field locally, then they will each precess at a different rate. The Larmor equation in the laboratory frame is given by:

$$
\omega=\gamma B_{0}+\gamma \Delta B
$$

where $\mathrm{B}_{0}$ is the static field and in the rotating frame:

$$
\omega=\gamma \Delta B
$$

The phase develops in time according to:

$$
\phi(t)=-\omega t=-\gamma \Delta B t
$$

As the spins become more and more out of phase, the signal rapidly vanishes and Equation B4.1.4 can be used to approximately estimate the rate of signal loss.

\section{The Spin Echo}

The breakthrough in early MR experiments came with the discovery of the spin echo. Rather than reading out the signal right after the $90^{\circ}$ pulse (which we will assume has been applied about the $x^{\prime}$-axis so the spins are tipped along the $+y^{\prime}$-axis), a second RF pulse is applied at a time $\tau$ later, a $180^{\circ}$ pulse, to refocus the spins (see Fig. B4.1.2). How this spin echo refocusing works is the topic of this subsection.

Consider the set of spins shown in Figure B4.1.2 all together at time $t=0$. As time progresses after the $90^{\circ}$ pulse but before the $180^{\circ}$ pulse, the spins continue to dephase. Now imagine that the $180^{\circ}$ pulse is applied about the $y^{\prime}$-axis so that at the time of the $180^{\circ}$ pulse all spins are flipped to the other side of the $y^{\prime}$-axis. Since the spins will continue to precess in the same direction as before (the $180^{\circ}$ pulse did not affect the local magnetic fields and, hence, the spins continue to rotate in the same direction and with the same rate as before), the spins will return to the $y^{\prime}$-axis at a time $\tau$ after the $180^{\circ}$ pulse or a time $2 \tau$ after the $90^{\circ}$ pulse. This coming together of all the spins that had run apart before the $180^{\circ}$ pulse is referred to as an echo, and in this case as the spin echo. It has often been compared to a group of runners, some fast and some slow, who after running a certain period of time stop and turn and run back toward the starting line at the same speeds at which they were running before. Of course, they all arrive back at the starting line together completely independently of the speeds with which they were running. The amazing thing about this sequence design is that any local field inhomogeneity is refocused with this method, making it a very powerful and robust approach to collecting data. The reason is similar to the runner analogy just given. The presence of the background inhomogeneous field causes the spins to all precess at different rates, just like the runners having different speeds. The $180^{\circ}$ pulse stops them and makes them run back toward the starting line, in this case, the $y^{\prime}$-axis. This rephasing is referred to as a spin echo or Hahn echo. 

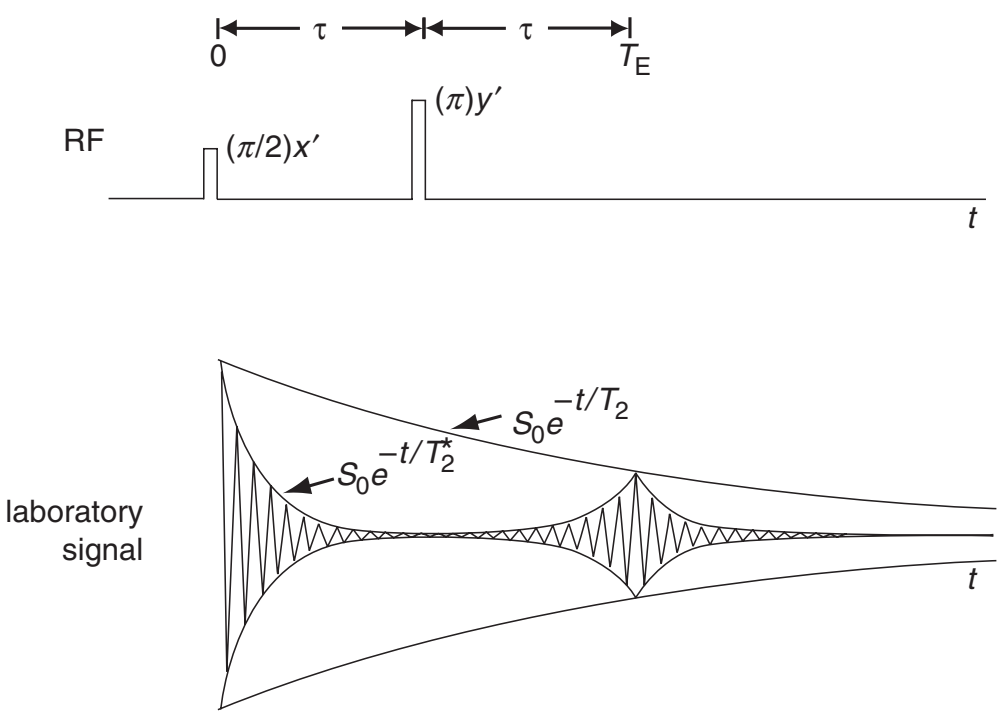

Figure B4.1.2 A $\pi / 2$ pulse is applied along the positive $x^{\prime}$ axis at $t=0$, and a $\pi$ pulse is applied along the positive $y^{\prime}$ axis at $t=\tau$ to invert the phase that the spins have accumulated. The spins then "rephase", producing what is called an "echo." Notice that the signal strength is still limited by the $T_{2}$ envelope at the echo time $T_{\mathrm{E}}=2 \pi$, as measured from the center of the RF pulse. The corresponding exponentials for the decay envelopes are found in Equation B4.1.24. Here, the $\pi$ pulse is drawn at twice the height, for the same width, as the $\pi / 2$ pulse, corresponding to the need for twice the $B_{1}$ field to get twice the angle of spin rotation. The echo shows a positive local maximum, instead of a negative local minimum, since the magnetization is rephased along $+y^{\prime}$. The subscripts on the RF pulse brackets denote the axis along which the RF field is applied.

\section{The Gradient Echo}

Let us go back to the assumption that the field is homogeneous. Now, we purposely add a background magnetic field variation in the form of a gradient along the $z$-axis-i.e.:

$$
B(z)=B_{0}+G \cdot z
$$

in the laboratory frame or

$$
B(z)=G \cdot z
$$

in the rotating frame. This gradient by itself would be a bad thing because we have just determined that its presence would cause signal loss from spin dephasing; however, if the gradient is applied first as a negative gradient and then as a positive gradient, (see Fig. B4.1.4) then the spins will refocus. Let us examine this more carefully. The negative gradient $-\mathrm{G}$ creates a counter-clockwise phase that grows with time $t$ according to:

$$
\phi(t)=\gamma G \cdot z \cdot t
$$

Now at a time $t_{2}$, if we turn off this gradient and reverse its polarity so that the new gradient is $+\mathrm{G}$, then for $t$ greater than $t_{3}$ the phase becomes: 


$$
\begin{aligned}
\phi(t) & =-\gamma G \cdot z\left(t-t_{3}\right)+\gamma G \cdot z\left(t_{2}-t_{1}\right) \\
& =-\gamma G \cdot z\left(t-t_{3}-t_{2}+t_{1}\right)
\end{aligned}
$$

which yields zero when $t=t_{3}+t_{2}-t_{1}$-i.e., all the spins are once again back in phase with each other.

In contrast to the spin echo, the gradient echo is formed through the application of a specially designed gradient rather than the application of a rephasing RF pulse; therefore, only one excitation pulse is required without the need of applying the $180^{\circ}$ pulse. Although both approaches are able to form an echo, the means through which the echo is formed is fundamentally different. This also leads to a difference in image quality as well as contrast.

\section{TECHNICAL DISCUSSION}

The goal of this unit is to study the signal behavior in the presence of background field inhomogenieties and introduce the concepts of spin echo and gradient echo sequence structures. The presence of a background field disrupts the ability to measure the signal because it causes spins to become out of phase with one another. The concept of an echo is introduced to bring the spins back into phase, at which time there would be a stronger signal to measure.

Under ideal conditions, the signal decays exponentially—i.e., in the rotating frame:

$$
S=S_{0} \exp \left(-t / T_{2}\right)
$$

When background field inhomogeneities are present, $T_{2}$ must be replaced by $T_{2}{ }^{*}$ so that the signal becomes:

$$
S=S_{0} \exp \left(-t / T_{2}^{*}\right)
$$

with:

$$
1 / T_{2}^{*}=1 / T_{2}+|\gamma \Delta B|
$$

where $\Delta B$ represents the local field inhomogeneity across a pixel. Although this extra signal loss is not generally exponential, the signal loss estimated from such an approximation is a good back-of-the-envelope calculation of the expected effect. The take home point is that the presence of the inhomogeneity causes the signal to decay faster. This approximation to the signal behavior is reasonable for random systems and takes on different forms for fixed background field variations. We shall not worry about those complications here. 


\section{The Spin Echo Method}

The spin echo sequence is based on the application of two RF pulses: a $90^{\circ}$ pulse followed by a $180^{\circ}$ pulse (or "refocusing" pulse). The plot showing the recovery of $T_{2}^{\prime}$ signal loss resulting from this sequence is shown in Figure B4.1.2. To understand the signal plot, we shall analyze three steps.

In the first step, it is generally sufficient to assume that the magnetization of a sample is tipped by the first pulse (the $90^{\circ}$ pulse) immediately into the transverse plane. Suppose this happens instantaneously at time $t=0$ such that, initially, the (excess) spins point along one of the main axes in the transverse plane. Subsequently, depending on the magnetic field that the spins experience, the spins at different positions begin to precess at different frequency, also referred to as dephase, relative to each other. The spatially varied magnetic field can be the applied gradient or, for now, the local magnetic field inhomogeneities. The accumulated phase of a spin at a spatial location $(r)$ in the rotating frame is found from Equation B4.1.8. The phase $\phi$ in this frame relative to the initial axis that spins aligned immediately after the application of RF pulse is:

$$
\phi(r, t)=-\gamma \Delta B(r) t \quad \text { for } 0<t<\tau
$$

where $\phi(r, 0)=0$ and $\tau$ is the time interval between the $90^{\circ}$ and $180^{\circ}$ pulses. Although we will focus on the spin echo at this point, this relationship between phase and magnetic field variation, $\Delta \mathrm{B}$, is of critical importance for the future discussion of gradient echo imaging methods and the effects of local magnetic field inhomogeneities in MR signal.

The entire second step, for present purposes, is considered to be instantaneous. Another RF pulse with twice the amplitude $\left(180^{\circ}\right)$, but otherwise identical to the first, is then applied. This is done to negate the phase of the originally accumulated phase. For example, the spins which had previously accumulated extra positive phase now have, at the instant after the $180^{\circ}$ pulse, the negative of that phase, and vice versa:

$$
\begin{aligned}
\phi\left(r, \tau^{+}\right) & =-\phi\left(r, \tau^{-}\right) \\
& =\gamma \Delta B(r) \tau
\end{aligned}
$$

where $\tau^{+}$and $\tau^{-}$denote the time immediately after and before the application of the $180^{\circ}$ pulse.

In the third step, the spins continue, after time $\tau$, to accumulate phase according to Equation B4.1.17:

$$
\begin{aligned}
\phi(r, t) & =\phi\left(r, \tau^{+}\right)-\gamma \Delta B(r)(t-\tau) \\
& =-\gamma \Delta B(r)(t-2 \tau) \\
& =-\gamma \Delta B(r)\left(t-T_{\mathrm{E}}\right)
\end{aligned}
$$

with the echo time defined by:

$$
T_{\mathrm{E}} \equiv 2 \tau
$$

Since the rate at which phase is accumulated by each spin is unchanged, all of the spins will return to $\phi=0$ at the same time, the echo time. Equation B4.1.18 shows that the accumulated phase of all spins experiencing a time-independent field variation will return 

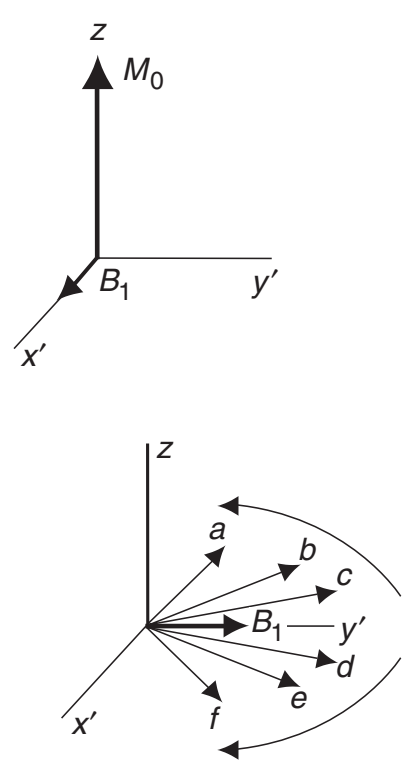
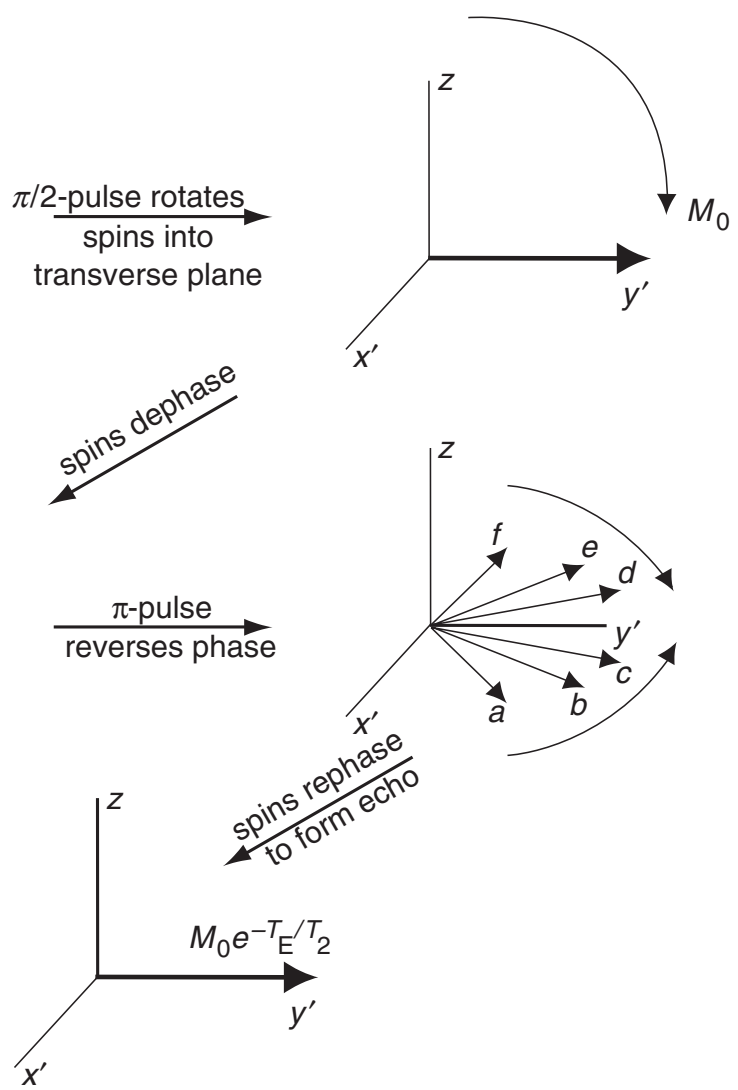

Figure B4.1.3 A simulation of an ensemble of spins in the rotating reference frame during a spin echo experiment. A $\pi / 2$ pulse rotates the spins, around the $x^{\prime}$ axis, into the transverse plane where they begin to precess. The spins accumulate extra phase, until this accumulation is inverted by the $\pi$ pulse. The spins continue to collect extra phase at the same rate and, at a later time, all spins return to the positive $+y^{\prime}$ axis together, forming an echo. The echo amplitude is still reduced, however, by the intrinsic $T_{2}$ decay.

to zero at $t=T_{\mathrm{E}}$, regardless of the value of $\Delta B$ and the position. Therefore, it is obvious that all of the spins will be realigned at the same time, and the realignment of the spins is called a spin echo, which is also referred to as a Hahn or RF echo. Figure B4.1.3 presents a simplified picture of the behavior of the individual spins during the experiment.

\section{Spin echo envelopes}

In this section, we will provide a detailed description regarding the signal envelopes of the spin echo formation between the $90^{\circ}$ and the $180^{\circ}$ pulses, between $180^{\circ}$ and the spin echo, and after the spin echo, respectively. This section is meant for more advanced users who are interested in further understanding of the signal behavior of the spin echo method and its relationship between $R_{2}, R_{2}{ }^{*}$, and $R_{2}{ }^{\prime}$.

The envelope of the transverse magnetization, $M_{\perp}(r, t)$, which is the main determinant of signal for the spin echo experiment, can be found by addressing the time dependence in the relaxation rate $R_{2}{ }^{S E}$ for the spin echo experiment. This is determined by the time rate of change in the rotating reference frame: 


$$
\left(\frac{d M_{\perp}}{d t}\right)^{\prime} \text { Relaxation }=-R_{2}^{S E} M_{\perp}
$$

where the primed represents that this notion is in the rotating frame. All other terms in the Bloch equation for the transverse magnetization are neglected. This equation is nothing more but a mathematical description of $T_{2}$ signal decay. It basically states that the rate of decay of transverse magnetization is determined by $T_{2}$ or $R_{2}$, which is commonly known as $T_{2}$ signal decay.

Before the $180^{\circ}$ pulse and after the echo, $R_{2}{ }^{S E}$ is the usual sum of extrinsic and intrinsic terms. Between the $180^{\circ}$ pulse and the echo, the extrinsic rate is negative, because the phase differences due to field inhomogeneities are being refocused. This corresponds to the change in sign implied by Equation B4.1.17. We have:

$$
R_{2}^{S E}(t)= \begin{cases}R_{2}^{\prime}+R_{2} & 0<t<\tau \\ -R_{2}^{\prime}+R_{2} & \tau<t<2 \tau=T_{\mathrm{E}} \\ R_{2}^{\prime}+R_{2} & t>2 \tau=T_{\mathrm{E}}\end{cases}
$$

to be compared with the simple free induction decay (FID) rate:

$$
R_{2}^{*}=R_{2}^{\prime}+R_{2}
$$

Within each interval, $R_{2}{ }^{S E}$ is constant, and the solution to Equation B4.1.20 has the usual exponential form:

$$
M_{\perp}(t)=M_{\perp}\left(t_{0}\right) e^{-\left(t-t_{0}\right) R_{2}^{S E}}
$$

Although any $t_{0}$ within the given interval can be taken as an initial time, the intention is to use Equation B4.1.23 to evolve the magnetization from the beginning of each interval $\left(t_{0}=0, \tau, T_{\mathrm{E}}\right.$, respectively).

Matching the exponential solutions of the separate differential equations at the two boundaries of the three intervals leads to the spin echo envelope (magnitude) for the whole experiment. We find:

$$
M_{\perp}(t)=M_{\perp}(0) \begin{cases}e^{-t / T_{2}^{*}} & 0<t<\tau \\ e^{-t / T_{2}} e^{-\left(T_{\mathrm{E}}-t\right) / T_{2}^{\prime}} & \tau<t<2 \tau=T_{\mathrm{E}} \\ e^{-t / T_{2}} e^{-\left(t-T_{\mathrm{E}}\right) / T_{2}^{\prime}}=e^{-t / T_{2}^{*}} e^{T_{\mathrm{E}} / T_{2}^{\prime}} & t>2 \tau=T_{\mathrm{E}}\end{cases}
$$

in terms of the decay times and the initial magnetization at $t=0$. The spatial dependence has been suppressed everywhere. The envelope shown in Figure B4.1.2 corresponds to Equation B4.1.24.

\section{Commentary on the spin echo}

The signal peaks at the echo, as seen in Figure B4.1.2, but it recovers only to the level dictated by the $T_{2}$ envelope. The spin echo does not reduce the effect of $T_{2}$ decay on the signal. The reason lies in the rapid time fluctuations in the intrinsic local fields. The inhomogeneities in these internal fields do not stay fixed in time. The rates at which phase 
is accumulated change with time, and, in general, no refocusing is possible. Fortunately, the irreversibility of the intrinsic $T_{2}$ loss is not a severe limitation for liquids $\left(T_{2}\right.$ is often three orders of magnitude shorter for solids). The time interval over which data are collected often can be made short compared with $T_{2}$.

Examples of other time-dependent spin effects include motion (macroscopic or microscopic) where the spins move from place to place during the experiment, such that they experience different fields at different locations. The spin echo will not refocus these types of inhomogeneities either.

Although the spin echo presents a way of reversing the effect of $T_{2}^{\prime}$, the recovery is complete only when $\phi(t)$ backtracks to zero for all of the spins. If the spins are not at this point, then the data will be tainted by $T_{2}^{\prime}$ decay, despite the effort to refocus the spins. For instance, in Figure B4.1.2, only the data point collected at $t=T_{\mathrm{E}}$ is completely refocused. The problem is acute when $B_{0}$ is rather inhomogeneous, since a very short $T_{2}^{\prime}$ implies a rapid drop-off in either direction away from the echo.

In addition, although the distinguishing feature of the spin-echo approach is refocusing dephase induced by the local magnetic field, there are, however, several major shortcomings associated with this approach from a practical point of view. First, the required two RF pulses make the repeat time $\left(T_{\mathrm{R}}\right)$ longer than the gradient echo sequences (see below for more details) which, in turn, implies a longer data acquisition time. This is particularly true for a $T_{2}$-weighted sequence that the $T_{\mathrm{R}}$ is normally on the order of $2 \mathrm{sec}$, making it difficult to employ a 3-D approach. Second, since one $90^{\circ}$ pulse and one $180^{\circ}$ pulse are required, the power deposition is, generally speaking, much higher than in the gradient echo approaches; therefore, the choice of imaging approach, whether either spin echo or gradient echo should be based upon the time allowed, the spatial resolution required and specific absorption rate (SAR) limits.

Almost all of the clinical protocols, regardless of the anatomy, have employed spin echo sequences. Different clinical protocols are given in detail in the clinical section of this manual. Some scenarios that could benefit from the application of utilizing a spin echo approach include those cases: where the effects of local susceptibility are anticipated to be large, when it is necessary to minimize the effects of local susceptibility, or when sensitivity to changes in $T_{2}$ is desired.

\section{The Gradient Echo Method}

In contrast to a spin echo approach, a gradient echo is formed through the application of gradients. Similar to those employed for the spin echo, phase arguments can be utilized to explain the formation of a gradient echo. Let us analyze the specific gradient sequence shown in Figure B4.1.4.

A constant negative gradient, $G_{z}=-G$ and $G>0$, is present in the time interval $\left(t_{1}, t_{2}\right)$. Based on Equation B4.1.25 and that a gradient can be defined as the changes of magnetic field as a function of distance, the phase accumulation due to the gradients for a spin at $z$, and at a time $t$, during the application of the first gradient lobe is:

$$
\phi_{G}(z, t)=\gamma G z\left(t-t_{1}\right) \quad t_{1}<t<t_{2}
$$

The sign is consistent with the counter-clockwise precession expected for a negative gradient and positive $z$-coordinate. 
A
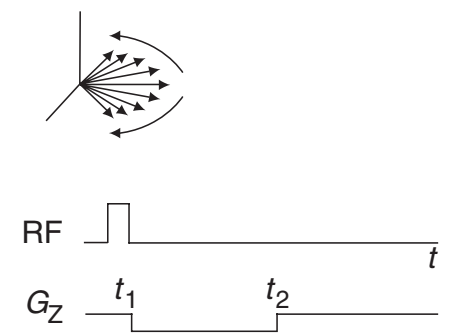

signal
B

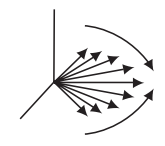

$$
t=0 \text {, }
$$

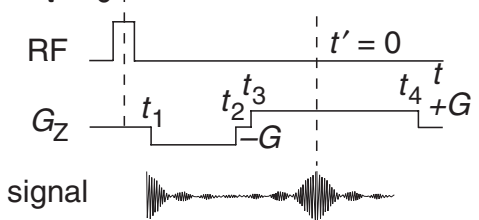

Figure B4.1.4 Immediately after the application of the RF pulse, a negative gradient is applied between $t_{1}$ and $t_{2}$. (A) The transverse magnetization starts to dephase owing to the magnetic field that spins experienced at different positions. (B) At $t=t_{3}$, a positive gradient is then applied to reverse the phase of the spins. At $t=t^{\prime}$, all spins will be refocused and a gradient echo is formed.

The second gradient lobe is positive $\left(G_{\mathrm{z}}=G\right.$ in terms of the same parameter $G$ ) during the time interval $\left(t_{3}, t_{4}\right)$. The phase behavior relative to the phase at the time origin in this interval is:

$$
\phi_{G}(z, t)=\gamma G z\left(t_{2}-t_{1}\right)-\gamma G z\left(t-t_{3}\right) \quad t_{3}<t<t_{4}
$$

Notice that there is no phase change during the period when the applied gradient is zero.

The gradient echo is now evident. The phase of Equation B4.1.26 returns to zero at:

$$
t=t_{3}+t_{2}-t_{1}
$$

for all $z$ and a gradient echo is formed.

The echo corresponds to that time during the second gradient lobe where the evolved area under the second lobe just cancels the area of the first lobe. The cancellation suggests the general condition for a gradient echo:

$$
\int_{t_{1}}^{t} G(t) d t=0
$$

i.e., the zeroth moment of $G(t)$ vanishes. The gradient echo analysis in imaging revolves around this criterion.

We have ignored the effects of local susceptibilities associated with the gradient echo method in the above discussion. In the presence of local susceptibility, $\Delta B$, additional phase term induced by $\Delta B$ will need to be added in Equations B4.1.25 and B4.1.26.

$$
\begin{array}{rlrl}
\phi(z, t)= & \gamma G z\left(t-t_{1}\right)-\gamma \Delta B t & t_{1}<t<t_{2} \\
\phi(z, t)= & \gamma G z\left(t_{2}-t_{1}\right)-\gamma G z\left(t-t_{3}\right)-\gamma \Delta B t_{2} & \\
& -\gamma \Delta B\left(t_{3}-t_{2}\right)-\gamma \Delta B\left(t-t_{3}\right) & & t_{3}<t<t_{4} \\
\phi\left(z, T_{E}\right) & =-\gamma \Delta B T_{\mathrm{E}} & & \text { when } t=t_{3}+t_{2}-t_{1} \equiv T_{\mathrm{E}}
\end{array}
$$


Even though the first two terms vanished when $t=t_{3}+t_{2}-t_{1}$, there still remains a phase dispersion which equals $-\gamma \Delta B T_{\mathrm{E}}$. Therefore, unlike a spin echo method, a local susceptibility induced phase shift will not be refocused by a gradient echo approach and the amount of phase dispersion will depend on both the local magnetic field as well as $T_{\mathrm{E}}$. As a result, a minimal $T_{\mathrm{E}}$ that can be achieved with the specifications of the MR scanner is normally utilized for a gradient echo experiment so that the effects of local susceptibility can be minimized.

\section{Commentary on the gradient echo}

As mentioned previously, one of the major fundamental discrepancies between the gradient echo and the spin echo is the means by which the echo is formed. With the spin echo approach, local susceptibility will be refocused at the echo, while a signal reduction is anticipated for the gradient echo owing to the unrecoverable dephasing induced by the local susceptibility. This has been the major limiting factor for the gradient echo approach. Specifically, signal reduction or signal void is commonly seen with gradient echo images where local susceptibility is large. Nevertheless, the ability to acquire images rapidly has offered major advantages in increasing the throughput of patients; therefore, almost all of the MR imaging protocols includes one or more gradient echo sequences. In addition, thanks to the ability to acquire images rapidly, 3-D acquisition is possible which in turn can provide high spatial resolution images without gaps between slices.

Some scenarios that could benefit from the application of the gradient echo approach include the cases where any of the following are desired: rapid contiguous slices, high resolution both in-plane and through-plane, or a high sensitivity to $T_{2}^{*}$ changes.

\section{KEY REFERENCES}

Haacke, E.M., Brown, R.W., Thompson, M.R., and Venkatesan, R. 1999. Magnetic Resonance Imaging: Physical Principles and Sequence Design. John Wiley \& Sons, New York.

This book covers the technical discussion here as well as other advanced materials in detail.

Hahn, E.L. 1950. Spin echoes. Phys. Rev. 80:580.

This paper introduces the fundamental concepts of spin echo.

Contributed by Weili Lin

University of North Carolina at Chapel Hill

Chapel Hill, North Carolina 